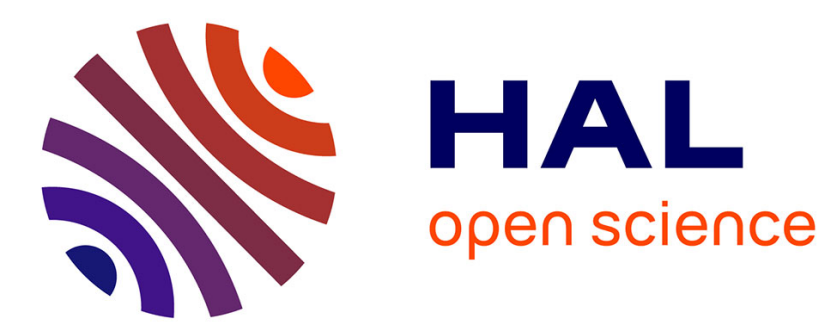

\title{
POLESAT : e-Atlas de Santé, Planification et Développement Durable
}

Anne Quesnel-Barbet, Julien Soula, Régis Beuscart

\section{To cite this version:}

Anne Quesnel-Barbet, Julien Soula, Régis Beuscart. POLESAT: e-Atlas de Santé, Planification et Développement Durable. Spatial Analysis and GEOmatics SAGEO '2009, Nov 2009, PARIS, France. , 2009. hal-01996431

\section{HAL Id: hal-01996431 https://hal.science/hal-01996431}

Submitted on 28 Jan 2019

HAL is a multi-disciplinary open access archive for the deposit and dissemination of scientific research documents, whether they are published or not. The documents may come from teaching and research institutions in France or abroad, or from public or private research centers.
L'archive ouverte pluridisciplinaire HAL, est destinée au dépôt et à la diffusion de documents scientifiques de niveau recherche, publiés ou non, émanant des établissements d'enseignement et de recherche français ou étrangers, des laboratoires publics ou privés. 
Introduction

La géographie de la santé est une discipine jeune, souvent non connue des
professionnels et du grand public

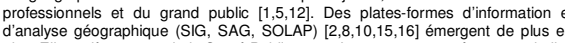

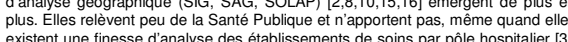

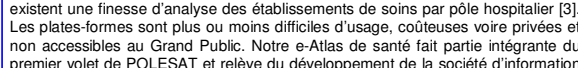

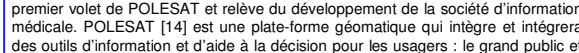

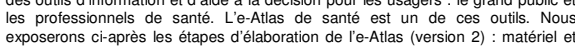

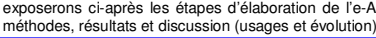

\section{Matériels et méthodes}

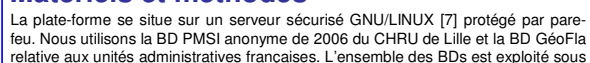
relative aux unités administrtatives françaises. L'ensemble des BDs est explotíe sous
SAS. La mise en page Web nécessite l'emploi de Jalbum et de 'Poutil d'Analyse

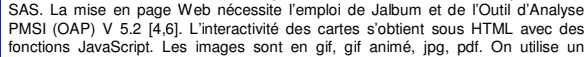
fonctions Javascript. Les images sont en gif, gif animé, jpg, pdf. On utilise un
formulaire HTML et un tratiement PHP pour leenvoi des remarques. Une alarm incident' est activée par l'outil de surveillance NAGIOS [9]

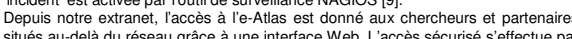
authentifications forte (certificat) et simple (.htaccess) [9]. Le protocole HTTPS

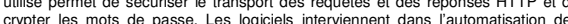
crypter les mots de passe. Les logiciels interviennent dans l'automatisation des
traitements de données (SAS) et la mise en formie des résultats (Jalbum)

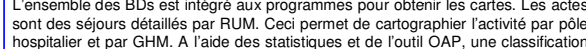

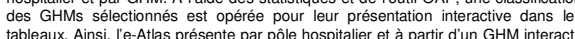
la carte et son commentaire. Linterprétatation de la carte s'effectue aidé des complementaires. Les
sous HTML. Les re

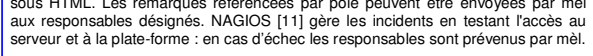

Figure 2: tableau partiel des Groupes Homogènes de Malade pour le pôle

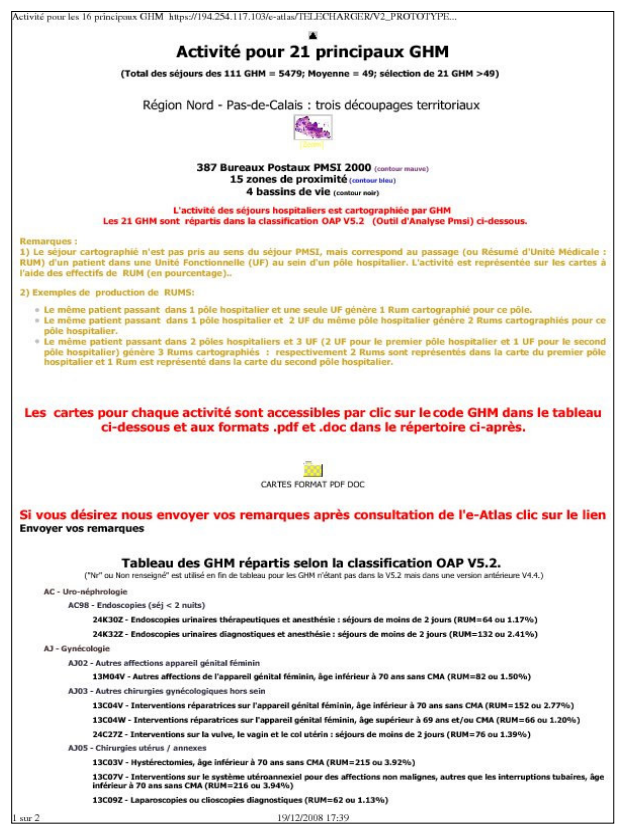

POLESAT : e-Atlas de Santé, Planification et Développement Durable
Figure 1:36 pôles hospitaliers fictifs, liens interactifs

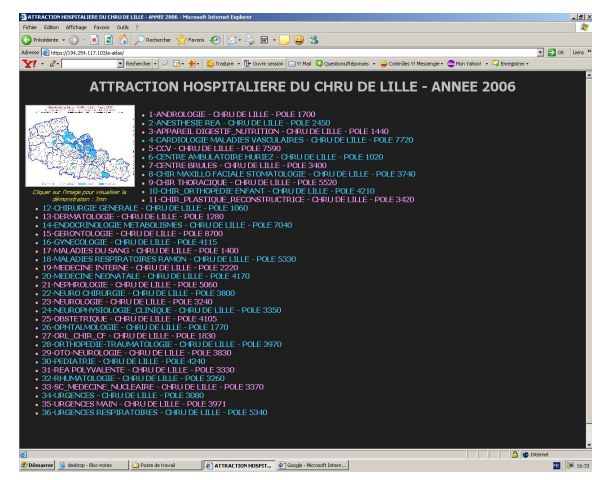

Figure 3: carte en plages de couleur, commentée, au format gif.

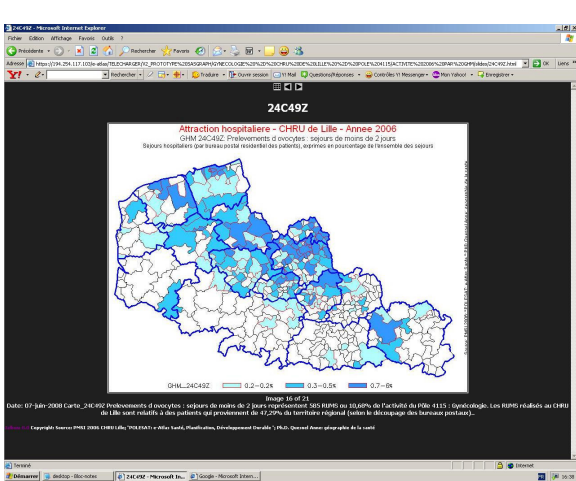

Figure 4: carte choroplèthe en couleur, avec infobulle (en jaune)

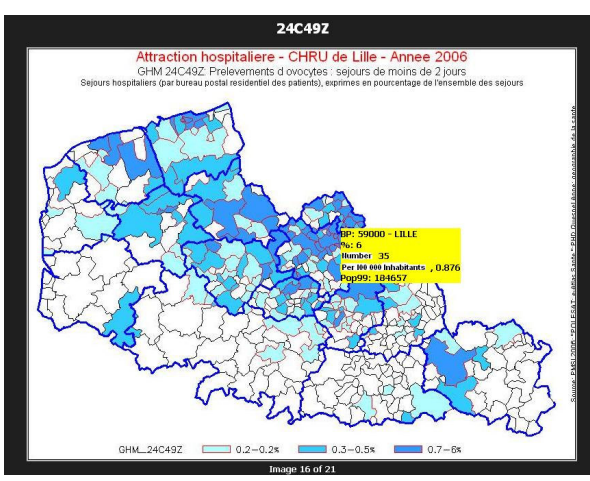

Résultats

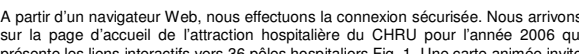
présente less liens interactifis vers 36 pôles hospitalierrs Fig. 1. Une carte animeée invite

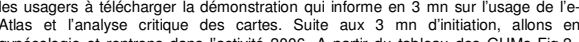

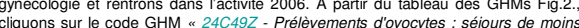

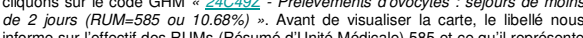

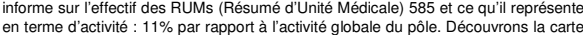

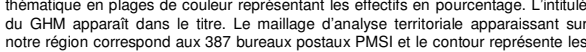

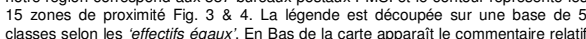
aux activités de séjours et attraction géographique des séjours $47,29 \%$. Le type
d'attraction (de proximite, de recours ou autre) s'evalue par rapport à ce pourcentage, à la forme de la distribution, au dépassement constaté ou non de
limites territoriales et au regard donne sur lensemble de la carte. Les usagers en

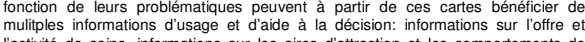
lactivité de soins, informations sur les aires d'attraction et les comportements de pratique des lieux, choix du service hospitalier, planification sanitaire (axes
et prospectif), auport de nouvelles connaissances, mémorisation spatiale etc.

\section{Discussion-conclusion}

L'e-Atlas permet létat des lieux des activités et de consommation de soins. Sa
fonction est d'apporter de nouvelles connaissances pour les usagers : I g grand public et les professionnels de santé. Les usages que lon peut en faire sont multiples et
répondront aux besoins des usagers. Voici un exemple d'usage : une dame vit à Maubeuge et envisage une prochaine intervention chirurgicale. Elle souhaite se
soigner à proximité du domicile lillois de ses entants pour faciliter les visites et être seule etou à l'aide de son médecin traitant, elle consultera lé-Atlas de santé. Le

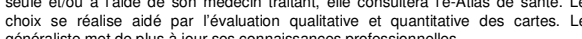
généraliste met de plus a jour ses connaissances profossionnelles.
Evolututions à venir : un premier lien vers l e serveur universitaire est prévu depuis Vintranet du CHRU de Lille. Notre tuture version ajoutera des cartes standardisées à bassins de vie ; un embellissement et des informations statistiques et administratives aux infobulles. L'exploitation des BDs 2006 portera sur I'ensemble des
établissements des secteurs public, privé et PSPH. La page d'accueil proposera en carte interactive des établissements par secteur qui redirigeront lusager par simple
clic vers l'atlas ad hoc. Nous Integrererons un $2 e$ outil de planification Polesat [13] :

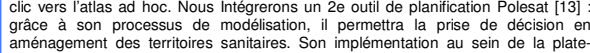

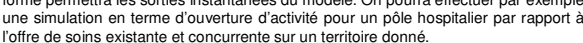

\section{Références}

1Amat-Rore.J.M. 2007. Climate change and tropical diseases: mobility systems

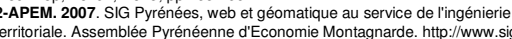

3-ARH. 2002. Parntage, Portail des Agences Régionales de l'Hospitalisation.

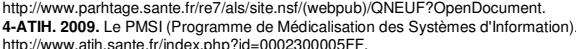

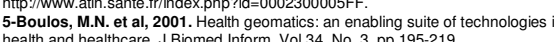

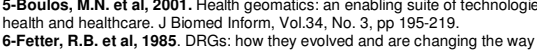
6-Fetter, R.B. et al, 1985. DRGS: how they evolved and are changing the way

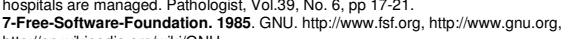

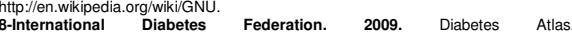

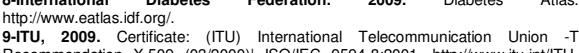

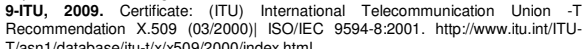

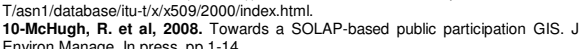

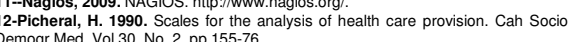
13-Quesnel - Barbet, A. 2007, A Mathematical model for hospital attraction area,
geography of spatial Utilization of the health services : a Newtonian modeling of

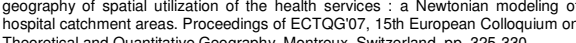
Theoretical and Quantitative Geography. Montreut, Sitzerland, pp. 325-330.
14-Quesnel - Barbet, A. et al, 2009, e-Atlas de sante, planification et develooppement durable. Proceedings of RiTS 2009 (Journées de Recherche en
magerie et technologies pour la a Santé). Lille, France, pp. 199-201. Calais.
16-Sloane, E. et al, 2005. Clinical engineering department strategic graphical
dashboard to enhance maintenance planning and asset management. Conf Proc
IEEE Eng Med Biol Soc, Vol 7 . pP $7107-7110$. 